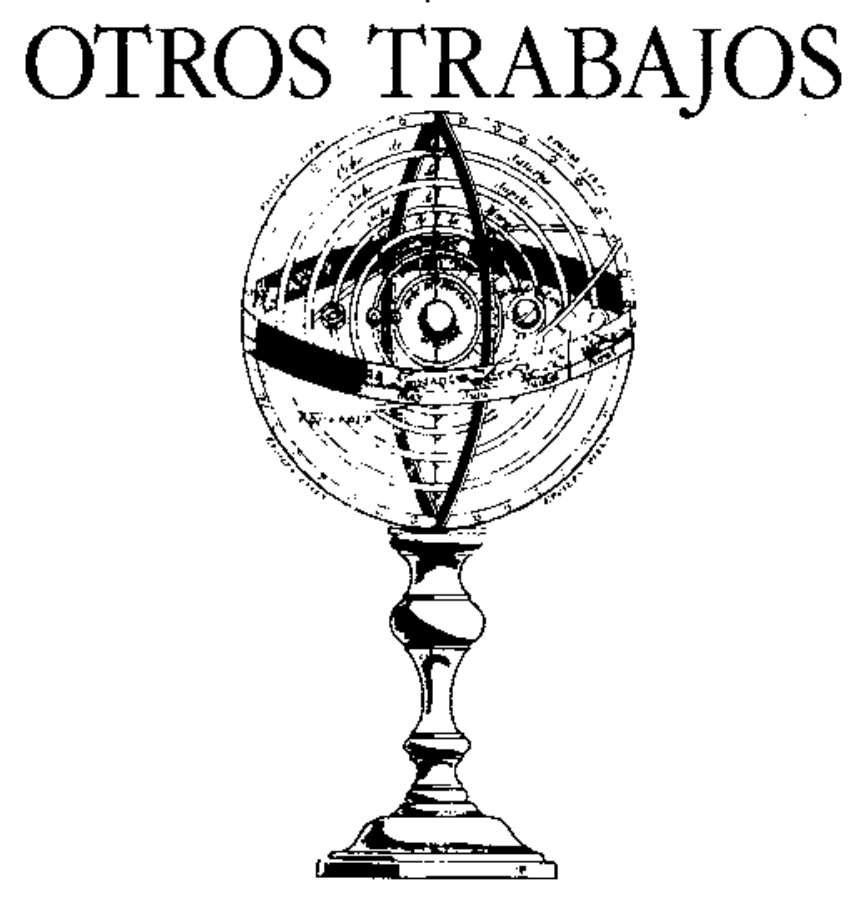

\title{
PSICOLOGÍA Y APRENDIZAJE DE LAS CIENCIAS. EL MODELO DE GAGNÉ
}

\section{GUTIÉRREZ, R.}

Departamento de Didáctica de las Ciencias. IEPS. Madrid.

\section{SUMMARY}

Gagné's model of learning is critically presented in this paper. Among others, the following aspects are included:

- Gagnés concept of learning.

- The criteria to establish learning sequencies.

- Those aspects of the model more frequently criticised by researchers.

- The follow-up trends of the model.

- Gagné's influence on classroom work.

\section{INTRODUCCIÓN}

En un artículo anterior (Gutiérrez 1987) describimos un método para detectar cuáles eran los problemas cen. trales relativos al aprendizaje de las Ciencias que se des- prendian del estudio de la literatura producjda en los últimos años en torno a esta área de investigación. Con este método descubrimos que eran cuatro los modelos 
de aprendizaje más utilizados: los de Ausubel, Gagné, Piaget y los inspirados en la Psicologia del Procesamiento de la Información. Y los temas que más parecían preocupar a los autores eran los siguientes:

a) El concepto de aprendizaje que se utiliza en los distintos modelos

b) los criterios de competencia para el aprendizaje que en los diversos modelos se establecen

c) los criterios para establecer secuencias de aprendizaje, dentro de la lógica interna del modelo

d) los aspectos del modelo que han recibido más criticas por parte de los investigadores

e) las líneas de evolución que ha seguido el modelo, desde su producción original hasta nuestro días.

En aquel artículo presentamos, desde esta perspectiva, el modelo de Ausubel. Ahora presentaremos, centrándonos en estos mismos puntos, el modelo de Gagné.

Como en la anterior publicación, siempre que sea posible utilizaremos citas literales de este autor, para mantener nuestra exposición en el nivel de objetividad posible, y en un intento de dejar a los lectores la posibilidad de contrastar sus opiniones, no solamente con nuestra propia interpretacion del modelo, sino con las palabras del mismo autor.

Utilizaremos la traducción al castellano de la primera edición del libro The conditions of Learning (1965), Las condiciones del aprendizaje (1971), mientras no se ad. vierta lo contrario.

\section{EL MODELO DE R.M. GAGNÉ}

\subsection{Concepto de aprendizaje}

En su libro Las condiciones del aprendizaje, Gagné (1971) sistematiza su pensamiento acerca de lo que es aprender. Tomamos de la Introducción del mismo (p.5) la siguiente definición:

El aprendizaje consiste en un cambio de la disposición o capacidad humanas, con carácter de relativa permanencia y que no es atribuible simplemente al proceso de desarrollo.

El cambio a que nos referimos se manifiesta como una modificación en la conducta y se puede inferir de la comparación del tipo de conducta observada por el individuo antes y después de ser colocado en la «situación de aprendizaje»" (1).

Seguidamente explica los términos de esta definición (p.6):

«1. En primer lugar, nos encontramos con el sujeto que aprende, que es un ser humano (...). Para nuestro propósito, las partes más importantes del sujeto son sus sentidos, su sistema nervioso central y sus músculos. Los sucesos que ocurren a su alrededor impresionan sus sentidos y dan lugar a cadenas de impulsos ner- viosos organizados por su sistema nervioso central, y especialmente por el cerebro. Esta actividad nerviosa llega a alterar el mismo proceso organizador: se dice entonces que el sujeto aprende.

2. El conjunto de sucesos que estimulan los sentidos se denomina situación estimulante (o situaciónestímulo). Estímulo es la denominación que recibe un suceso aislado.

3. La acción que resulta de la estimulación y subsecuente actividad nerviosa se llama respuestay (1).

$\mathrm{Y}$ aclara los conceptos que está introduciendo con un ejemplo muy sencillo, como aprende un niño el nombre del cartero (p. 7):

"Al principio, el niño no dice señor Tal cuando ve al cartero; simplemente le llama "hombre» o lo señala. Suponiendo que el niño tiene cierta capacidad verbal, la situación estimulante podría consistir simplemente en la siguiente frase de su padre: 'Éste es el sentor Tal'. La respuesta piovocada en el niño es 'señor Tal'.

Cuando de nuevo ve al cartero, el niño vuelve a decir: 'señor Tal'. En este momento se puede sacar la conclusion de que el nino ha aprendido, se ha producido un cambio en su actuación, ha adquirido una nueva capacidad».

Más adelante (cap. II, pp. 28-29) explica más su pensamiento a este respecto:

"La situación estimulante se encuentra, por lo general fuera del sujeto (...) y puede identificarse y describirse en términos físicos $(. .$.$) .$

El resultado es también, en realidad, exterior, por lo general fuera de sujeto (...) y puede identificarse y describirse en términos físicos (...).

El resultado es también, en realidad, exterior al sujeto. Consiste en una respuesta, $R$, o en una serie de respuestas cuya consecuencia es un producto identificable (...).

La naturaleza de la conexión entre $\mathrm{E}$ y $\mathrm{R}$ no puede observarse directamente. Los estudios sobre el funcionamiento del sistema nervioso podrán algún día proporcionar una mayor comprensión de dichos mecanismos (...)». (1)

Gagné advierte en el libro que veniamos citando, que su interés fundamental no es el aprendizaje, sino las condiciones del aprendizaje. Sin embargo, su concepto de lo que es aprender necesariamente establece un filtro muy específico para describir dichas condiciones, como se puede deducir de sus palabras (p. 20):

"Las capacidades previas del sujeto ejercen un importante papel en la determinación de las condiciones requeridas para un aprendizaje posterior (...).

Al conjunto de capacidades previas poseidas por el sujeto se le puede aplicar la denominación de condiciones internas al sujeto. Pero además hay un segunda ca- 
tegoria de condiciones que son exteriores al sujeto, e independientes en su acción (...). La adquisición de capacidades distintas requieren no sólo diferentes capacidades previas, sino también diferentes condiciones externas». (1)

A este respecto, la posición que mantiene Gagné es la siguiente:

«Hay tantos tipos de aprendizaje como condiciones características para el mismo. Podemos diferenciar dichos tipos mediante la descripción de los factores que integran las condiciones de aprendizaje en cada caso. $\mathrm{Pa}$ ra su investigación e identificación hay que tener en cuenta, primero las condiciones internas del sujeto y, después, la situación estimulante externa al mismo. Cada tipo de aprendizaje arranca de una capacidad interna distinta, y generalmente exige también diferentes situaciones externas. Los prototipos de aprendizajes útiles son los que se perfilan mediante esta descripcion de sus condiciones) (p. 20-21).

Según estas premisas, Gagné describe minuciosamente en el libro que venimos citando ocho tipos distintos de aprendizaje, resumiendo sus caracteristicas de la siguiente manera (p. 54 y 55 ):

« ${ }^{\circ}$. Reacción ante una señal: el individuo aprende a dar una respuesta amplia y difusa ante una señal. Se trata de la clásica respuesta condicionada de Paulov (1927).

$2^{\circ}$. Estimulo-respuesta: el sujeto adquiere una respuesta precisa ante un estímulo discriminado. Lo aprendido es una conexión (Thorndike 1898) o una operante discriminada (Skinner 1938), algunas veces llamada respuesta instrumental (...).

$3^{\circ}$. Encadenamiento: lo que se adquiere es una cadena formada por dos o más conexiones del tipo $\mathrm{Ee} \rightarrow \mathrm{R}$. Las condiciones para tal aprendizaje han sido descritas por Skinner (1938) y otros (...).

$4^{\circ}$. Asociación verbal: es el aprendizaje de cadenas verbales. Básicamente, las condiciones son semejantes a las otras cadenas (de tipo motor). Sin embargo, la presencia del lenguaje en el hombre la convierte en un $\mathrm{t}-$ po especial porque los eslabones internos pueden seleccionarse entre el repertorio lingüistico previamente aprendido por el individuo (...).

$5^{\circ}$. Discriminación múltiple: el individuo aprende a dar cierto número (n) de respuestas identificadoras distintas ante otros tantos estimulos diferentes, los cuales pueden parecerse unos a otros en su apariencia externa en mayor o menor grado. Aunque el aprendizaje de cada conexión $\mathrm{Ee} \rightarrow \mathrm{R}$ se puede encuadrar en el segundo tipo, unas conexiones tienden a interferir en la retención de las otras, y viceversa (...).

$6^{\circ}$. Aprendizaje de conceptos: el sujeto adquiere la capacidad de dar una respuesta común a una clase de estímulos que pueden diferir ampliamente unos de otros en cuanto a su aspecto externo. El sujeto puede, en es- te caso, dar una respuesta que identifique una clase completa de objetos o fenómenos (...).

$7^{\circ}$. Aprendizaje de principios: en términos muy sencillos, un principio es una cadena de dos o más concep. tos. Controla la conducta en la forma sugerida por la regla verbal del tipo: 'Si $\mathrm{A}$, entonces $\mathbf{B}$ ', la cual, por supuesto, puede ser aprendida según el tipo 4 .

$8^{\circ}$. Resolución de problemas: es una clase de aprendizaje que requiere del razonamiento. Dos o más principios anteriormente aprendidos se combinan ahora de forma que se produce una nueva capacidad, que parece depender de un principio 'de orden superior'.

Gagné advierte (p. 53) que aunque los tipos de aprendizaje que él describe sean ocho, no quiere decir que este número sea definitivo:

"¿Podrían ser siete, nueve o diez, en lugar de ocho? Desde luego. Puesto que la investigación continua, es posible que sea necesario establecer formulaciones nuevas de dichas condiciones, desglosar algunas o-lo que parece menos probable- eliminar otras».

Para comprender la concepcion gagnetiana del aprendizaje hay que detenerse en otro punto esencial de su pensamiento: su concepción jerárquica. Pensamos que en este punto también es interesante dejar que sea él mismo quien explique este concepto (p. 55-56):

«La condición más importante que permite distinguir entre una forma y otra de aprendizaje es su estado inicial, en otras palabras: sus requisitos previos. Las condiciones necesarias para realizar un encadenamiento requieren que el individuo haya aprendido previamente las conexiones del tipo $\mathrm{Ee} \rightarrow \mathrm{R}$ que le sean posibles, con objeto de que puedan ser relacionadas. Si no se cumple esta condición nos vemos obligados a trabajar con condiciones que permitan el establecimiento de dichos requisitos (...). Esta generación aplicada a las variedades de aprendizaje que hemos estudiado, se puede expresar brevemente de la forma siguiente:

Resolución de problemas (tipo 8) supone como requisitos previos los principios (tipo 7), que a su vez supo. nen como requisitos previos los conceptos (tipo 6), que suponen como requisitos previos las discriminaciones múltiples (tipo 5), que suponen como requisitos previos las asociaciones verbales (tipo 4) o cadenas de otra clase (tipo 3), que suponen como requisitos previos las conexiones $\mathrm{Ee} \rightarrow \mathrm{R}$ (tipo 2).

Cabría agregar (...) que las conexiones $\mathrm{Ee} \rightarrow \mathrm{R}$ (tipo 2) necesifan del aprendizaje de seffales (tipo 1) como requisito previo. Esto puede ser cierto, pero no parece deducirse con seguridad de las pruebas con que contamos hasta el presente (...)».

\subsection{Criterios de competencia para el aprendizaje}

Según hemos visto anteriormente, el sujeto que aprende parte del dominio de una capacidad interna determinada, se le coloca en una sítuación de aprendizaje 
(estimulos externos) y se espera que el producto sea otra conducta externa que demuestre la adquisición de una capacidad de orden superior. Cuando esto sucede así decimos que el sujeto ha aprendido.

Las capacidades del sujeto, de acuerdo con las anteriores afirmaciones de Gagné, se fundamentan unas sobre otras. De tal manera que adquirir un determinado aprendizaje, por ejemplo, un concepto (tipo 6), supone que el sujeto ha adquirido anteriormente las capacidades previas que son requisitos necesarios para que el aprendizaje se produzca. Es decir, es capaz de hacer discriminaciones múltiples (tipo 5 ) en relación a este concepto; lo que supone el aprendizaje previo de las asociaciones verbales correspondientes (tipo 4); lo que supone, a su vez, la capacidad para establecer encadenamiento (tipo 3), etc. Es decir: el criterio de competencia para la adquisición de un aprendizaje determinado consiste en que el sujeto haya adquirido previamente los tipos de aprendizajes que estén situados por debajo del mismo en la correspondiente jerarquía.

¿Y cómo se determina esa jerarquia de aprendizaje para la adquisición de una determinada capacidad? De nuevo hay que decir que es el mismo Gagné el que mejor explica su propio pensamiento a este respecto (pp. 157-158):

«Si la adquisición de determinadas capacidades se fundamenta en la posesión de otras, es posible entonces 'actuar hacia atrás' respecto a cualquier objetivo de aprendizaje necesario como requisito previo; incluso es posible recorrer hacia atrás todo el camino hasta llegar a las asociaciones verbales y cadenas más sencillas. $\mathrm{Al}$ efectuar un análisis de este tipo se obtiene una especie de plano de lo que ha de aprenderse (...)».

En relación al sujeto mismo del aprendizaje (el alumno, en nuestro caso) Gagné señala (p. I59):

«Es importante advertir que no se pretende describir (con jerarquías de aprendizaje) una «secuencia evolutivas, relacionando el aprendizaje de un sujeto con su estado de desarrollo o edad cronológica. Los tipos simples de aprendizaje tienen lugar durante todo el tiempo en que el individuo es sujeto de aprendizaje, cualquiera que sea su edad. Un estudiante de cálculo, por ejemplo, aprende a identificar el signo de integración esencialmente bajo las mismas condiciones en que otro de aritmética aprende el signo de dividir. En ambos casos se puede comprobar una secuencia de aprendizaje en la que las formas más complejas de aprendizaje se fundamentan en las más simples. Es este tipo de secuencia el foco de nuestro interés, independientemente del estado de desarrollo del sujeto (...). El individuo aprende primero las cosas más sencillas; a continuación más complejas cada vez; a medida que esto ocurre el sujeto se va haciendo mayor».

Parece que con estos párrafos queda suficientemente claro que en este modelo el criterio de competencia para el aprendizaje de un determinado sujeto es independien- te de su edad y dependiente de su situación en una posible jerarquía de aprendizaje.

\subsection{Criterios para establecer secuencias de aprendizaje}

Si Gagné plantea un modelo jerárquico para el aprendizaje, cabría pensar que la forma de plantear las secuencias instructivas debe ser elemento fuerte de su teoría. Y así es, en efecto: la mayor parte de las investigaciones que se han hecho en el aula siguiendo el modelo gagnetiano han versado sobre la elaboración y valida. ción de jerarquias de aprendizaje.

En un artículo anterior al libro que venimos citando, titulado The acquisition of knowledge, Gagné (1962) describe de forma operativa el modo de elaborar jerarquias de aprendizaje. Traducimos de sus palabras en este artículo (p. 358):

«Cuando uno empieza a describir la ejecución de una clase determinada de tareas como criterio de conducta terminal, es posible identificar los conjuntos de aprendizajes subordinados requeridos (...). La pregunta puede ser expresada más exactamente de la siguiente manera: ¿qué tendría que ser capaz de hacer el individuo para poder realizar con éxito esta tarea, suponiendo que sólo se le van a dar instrucciones? Esta pregunta se aplica sucesivamente a las clases de tareas subordinadas que se han identificado en la respuestass.

Para utilizar esta técrica Gagné supone que el diseñador conoce la estructura de la materia que va a planificar, de manera que pueda identificar cada contenido de aprendizaje y los contenidos de aprendizaje subordinados de los que depende. Asi puede ir hacia atrás (Gagné 1971, p. 157) hasta obtener todo el "plano» del proceso, que va a ser el que guíe la secuencia de actuaciones en el aula (Gagné 1971 , p. 217 y ss.). La figura 1 es una muestra explícita de cómo puede realizarse este proceso.

Estas jerarquías elaboradas siguiendo la estructura de la Ciencia necesitan de una validación posterior. Pero hacen posible, en palabras de Gagné, «postular las relaciones entre el contenido de la instrucción y los requerimientos de las condiciones del aprendizaje» (1971, p. 159).

En la validación de las jerarquias habria que comprobar si se cumplen los supuestos en que se fundan, entre los que habria que destacar como más importantes:

$1^{\circ}$ Los individuos que demuestren competencia de aprendizaje en un determinado nivel deberán estar preparados para el aprendizaje de un nivel inmediatamente superior conectado con el anterior (Transferencia vertical).

$2^{\circ}$ Los individuos que son capaces de demostrar competencia de aprendizaje en un determinado nivel deben ser capaces también de demostrar competencia en los aprendizajes subordinados que aparecen en ta jerarquía. 
Otro punto al que Gagné concede mucha importancia, para evitar ambigüedades en el enunciado mismo de los elementos de la jerarquía, es que el comportamiento final que expresa el aprendizaje adquirido esté redactado en términos de "objetivos de conducta» (Gag.

figura $I$

Jerarquía para el aprendizaje de los principios de la hidrólisis de sales, propuesta por Gagné en su libro «Las condiciones del aprendizaje», p. 170.

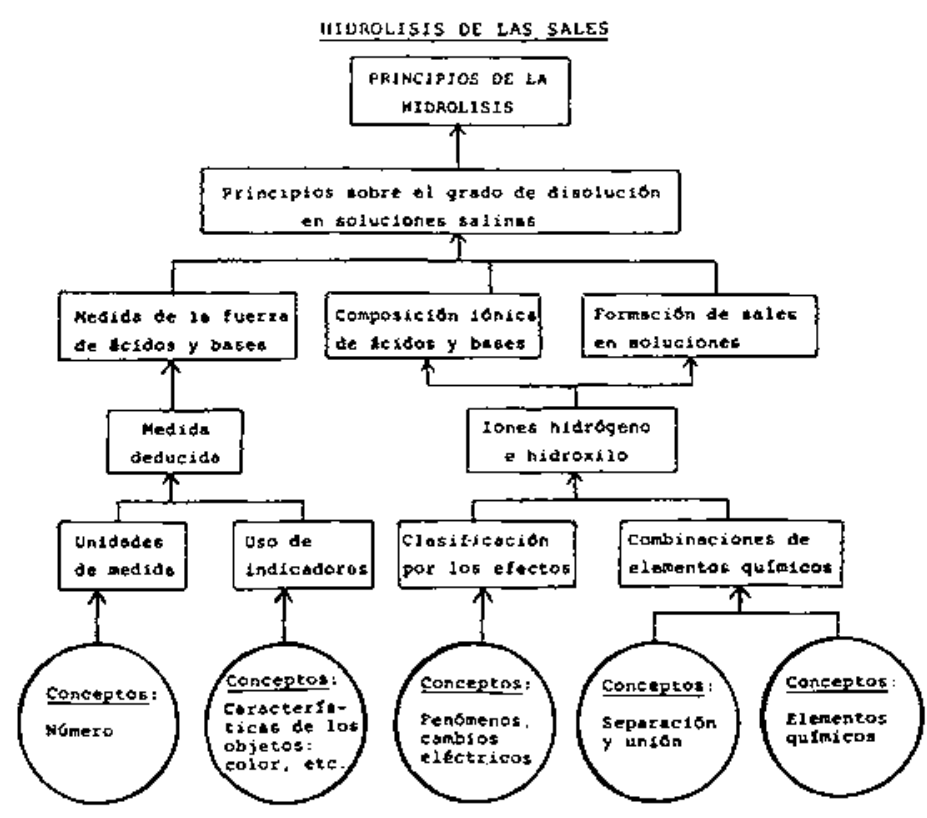

figura 2

En esta jerarquia cada tipo de aprendizaje (TA) está enunciado en forma de objetivos de conducta. Se define el nivel de entrada (habilidades ya supuestas) y se indica la secuencia didáctica que ha de seguirse en el aula (números exteriores en los recuadros). No se senala con flechas el sentido de recorrido de la jerarquia de prerrequisitos. Se da por supuesto que todos los unidos por líneas verticales se recorren preceptivamente de abajo arriba (según Briggs, 1973b, p. 96).

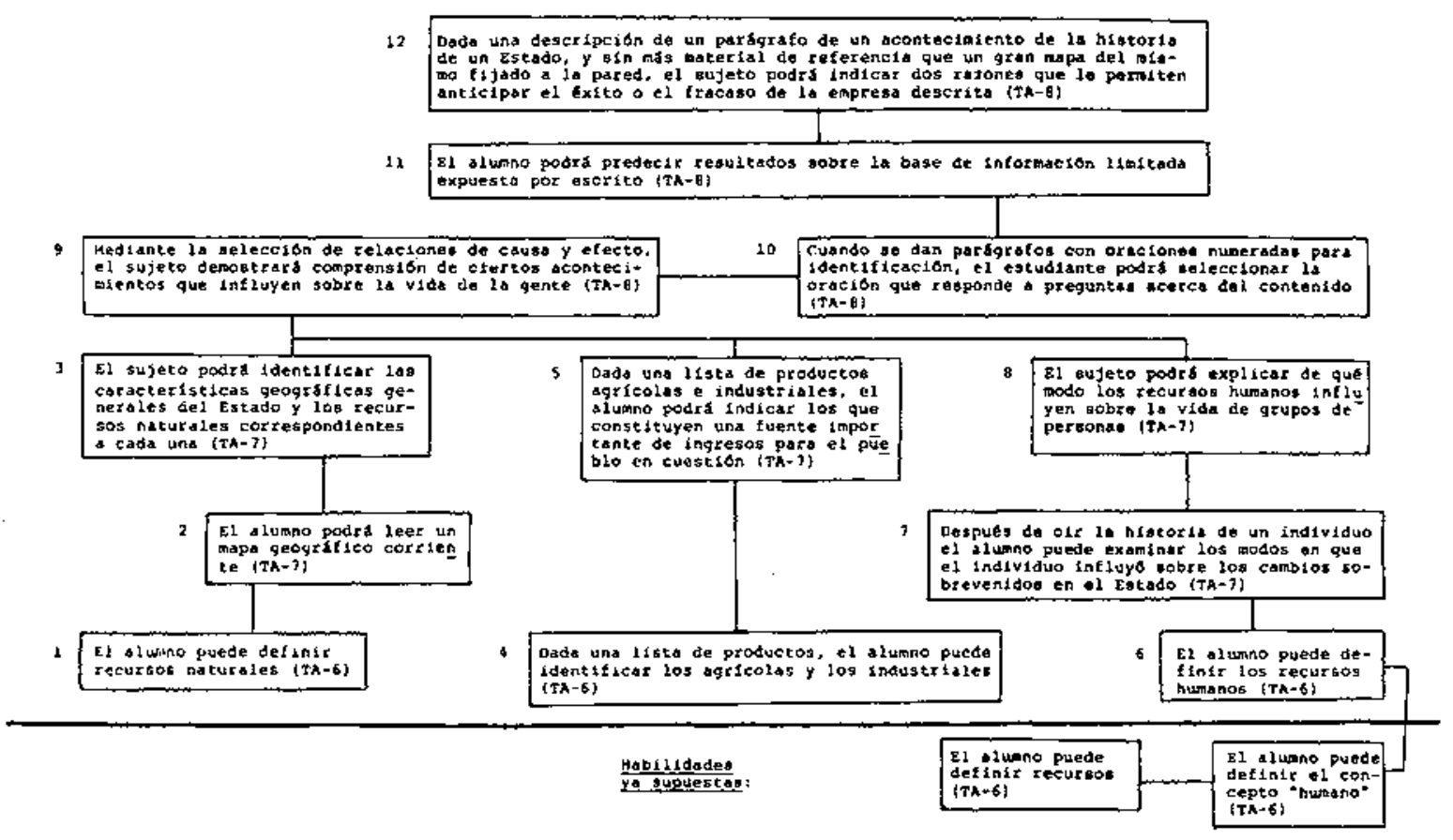




\section{ASPECTOS MÁS CRITICADOS DEL MODELO}

En las criticas realizadas al modelo gagnetiano de aprendizaje pueden destacarse tres aspectos que consideramos los más interesantes:

a) concepción epistemológica que informa al modelo

b) validación del mismo

c) ámbito de aplicación.

\subsection{Concepción epistemológica}

En cuanto a teórico del aprendizaje, a Gagné se le califica abiertamente como conductista por algunos autores (Pozo 1987, Driver 1982, Soulsby 1975, Phillips y Kelly 1975). Y a su teoría como «una teoría sobre cambio conductual, no sobre aprendizaje" (Soulsby 1975). Por consiguiente, no es de extrañar que desde el punto de vista epistemologico haya coincidencia en calificar la concepción del aprendizaje que tiene Gagné como empiricista-induccionista (Finley 1983, Cawthron y Rowell 1978). Y que se considere que este modelo sólo contempla la ciencia desde la perspectiva de la «ciencia normal», en el sentido en que Kuhn usa este término (Driver 1982).

En relación a este aspecto quizá la crítica más fuerte al modelo gagnetiano haya sido la realizada por Soulsby (1975), para el que dicho modelo sólo tiene un valor axiomático, «comparable al que tiene la geometría de Euclides", internamente consistente, pero no necesariamente conectada con el mundo real.

\subsection{Validación del modelo}

Un punto muy conflictivo en cuanto a la validación de la teoria de Gagné es la falta de consistencia que muestran las investigaciones al tocar el terna de la transferencia vertical del aprendizaje (Briggs 1973a, White 1974, Case y Bereiter 1984). En este sentido el modelo «jerárquico acumulativo" de Gagné dista mucho de cubrir la expectativa de que un nuevo aprendizaje superior sea construido por combinación de los inmediatamente inferiores ya adquiridos (Resnick 1983, Phillips y Kelly 1975). Y por otra parte también se muestra insuficiente para explicar el cambio conceptual que supone un nuevo aprendizaje (Driver 1982, Hewson 1981, Posner y otros 1982, Pozo 1987).

Para algunos autores, el modo en que se construyen las jerarquias (derivadas del análisis de la estructura del contenido) supone el establecimiento de prerrequisitos lógicos (West y Fensham 1974, Phillips y Kelly 1975, Novak 1982, Tomlinson 1984) que pueden ser significativos para el experto en la disciplina, pero que no tienen por qué serlo para el que no la conoce (Hartley y Davis 1976). Esta posición incluso lleva al absurdo de hacer inútil el planteamiento de la validación de las jerarquías de aprendizaje, ya que los prerrequisitos lógicos son necesarios (Phillips y Kelly 1975). Por otra parte, esto supone que la secuencia psicológica que si- gue el sujeto que aprende se subordina a la secuencia logica del concepto, al disenar la jerarquía, lo cual es un «a priori» que elimina las diferencias individuales de los alumnos y no coincide con el modo en que estos aprenden (Posner y Strike 1976, Soulsby 1975, White 1974, Raven 1966-67, 1972, Bass y Montague 1972). En este sentido Phillips y Kelly (1975) llegan a afirmar (p. 362):

"La existencia de las jerarquías gagnetianas no es un hecho que haya que buscar en el dominio de la Psicología. No se van a descubrir de modo experimental, sino analizando los contenidos de las materias, como matemáticas o física. Llevar a cabo el diseño de jerarquías gagnetianas necesita de las habilidades y el conocimiento de un físico o un matemático, más que de aquélias que posee un psicólogo» (2).

\subsection{Ambito de aplicación del modelo gagnetiano}

Prácticamente, todos los autores que han hecho una crítica a la teoria de Gagné coinciden en restringir sus áreas de aplicación a la enseñanza programada o cam. pos afines, con poca complejidad intelectual (Soulsby 1975, Driver 1982, Jones y Russel 1979, Hartley y Davis 1976). El hecho de la insistencia en enunciar los aprendizajes en términos de objetivos de conducta, parece excluir de esta manera de entender el aprendizaje, ámbitos tan importantes como el afectivo, el estético, el valoral, etc.

En todo caso, y a pesar de la identificacion que hace Gagné en algunas de sus publicaciones (Gagné 1985, Gagné y Dick 1983) entre las habilidades intelectuales y el conocimiento de tipo procedimental, parece claro que este modelo no es adecuado para este tipo de aprendizaje, sino fundamentalmente para los de tipo declarativo (3).

Por tultimo, hay autores que advierten de la posibilidad de que utilizando este modelo se consiga un éxito en el aprendizaje que, a pesar de significar un determinado dominio de las destrezas intelectuales, no garantice la comprensión de lo aprendido (realización de tareas que se llevan a cabo sabiendo el cómo pero no el por que (Kellet, West y Woodruff 1977).

\section{TENDENCIAS QUE SE OBSERVAN EN LA EVOLUCIÓN DEL MODELO}

A diferencia del modelo ausbeliano, en el que las líneas de evolución han sido dirigidas principalmente por los seguidores del mismo (Gutiérrez 1987), en este caso es el propio Gagné el que marca la pauta de su pro. pia evolución. Como veremos a continuación, su modelo conceptual se va enriqueciendo paulatinamente, a medida que incorpora datos de investigaciones provenientes de campos afines a su propia ideología científica (Novak 1982). Pero su concepto de aprendizaje conserva las caracteristicas descritas en su obra de 1965 
(ed. castellana 1971). Y sin duda ninguna, sus implicaciones didácticas se mantienen idénticas en cuanto a la aplicación que del modelo se realiza en el aula (Case y Bereiter 1984, Nesbit y Hunka 1987, Olarewaju 1987). Pero veamos cóno se produce esta evolución.

En 1974 Gagné y Briggs publican Principles of instructional design (en castellano La planificación de la enseñanza, 1976). En este libro se observa un cierto cambio en el lenguaje (se empieza a hablar de información de modo muy sustancial, de almacenamiento en la memoria del estudiante, de procesamiento mental) que recuerda al utilizado en las teorías del Procesamiento de la Información. Al estudiar el aprendizaje se amplía el marco de referencia, según puede verse en la figura 3 , y se describen ucinco categorías de resultados del aprendizaje», o "capacidades humanas": habilidades intelectuales, estrategias cognitivas, información verbal, destrezas motoras y actitudes (Gagné y Briggs 1976, p. 38). Pero toda la planificación de la enseñanza que se hace en el libro está basada en un concepto del aprendizaje semejante al descrito en Las condiciones del aprendizaje, y en una aplicación a la instrucción de las habilidades intelectuales idénticas a las descritas en 1965 (figura 4).

También en 1974 (aunque con posterioridad al anterior) publica Gagné otro libro Ensential of learning for-

figura 3

Nueve anos más tarde de la publicación de la primera edición de su libro "Las condiciones del aprendizaje», Gagné amplia su marco de referencia al explicar el fenómeno del aprendizaje. Esta figura es expresiva de elho (según Gagné y Briggs, 1976, p. 21).

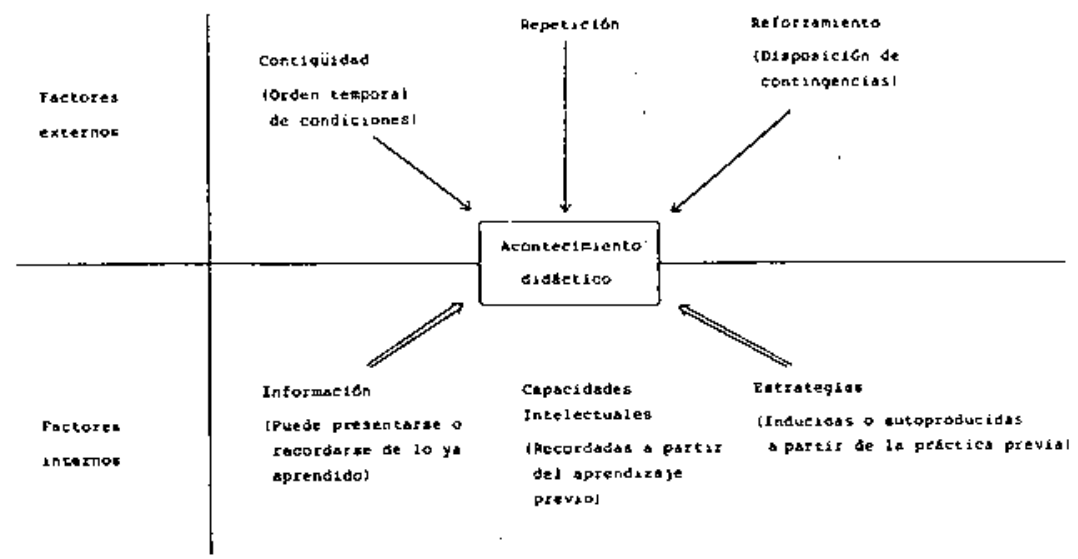

figura 4

A pesar del amplio marco de referencia que se describe en la figura 3, no se avanza al planificar la enserianza más allá de lo que se dice cn "Las condiciones del aprendizaje». La figura 4 muestra el eje vertebrador del pensamiento didáctico que se desarrolla en el libro, poco diferente del explicitado en 1965 (según Gagné y Briggs, 1976, p. 51).

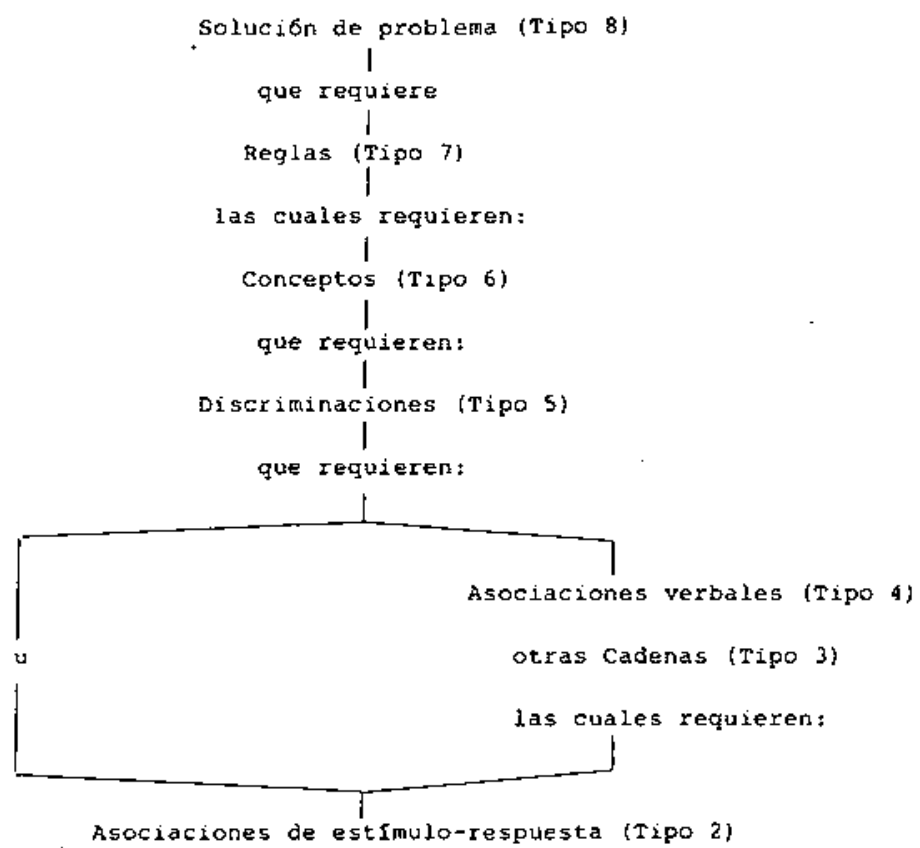


instruction (en castellano Principios básicos de aprendizaje para la instrucción. 1975) donde ya de manera abierta presenta un modelo para el aprendizaje tomado de la teoría del Procesamiento de la Información (Prefacio, p.5). El modelo que ofrece y los teóricos que cita no dejan lugar a dudas en este punto (figura 5). Sigue categorizando los resultados del aprendizaje en los cinco tipos descritos en el libro anterior, (Gagné y Briggs 1976), aunque de las "habilidades intelectuales» sólo describe las discriminaciones, los conceptos, las reglas y las reglas de orden superior. Quizá la novedad más fuerte que presenta esta obra es la dedicación de un capitulo a los procesos de aprendizaje, que describe en términos tomados de la psicología del procesamiento de la información y que define como actividades internas al sujeto que tienen lugar en su sistema neryioso central. En la tercera edición del libro Las condiciones del aprendizaje, realizada en 1979, se recogen y se amplian los avances apuntados en las dos obras anteriores (Gagné y Briggs 1976, Gagné 1975), pero no se aportan novedades importantes (Prefacio de la $3^{\mathbf{a}}$ edición).
Quizá la publicación que signifique más esfuerzo teórico por parte de Gagné en la sistematización de la evolución de su pensamiento en relación al aprendizaje, sea el artículo publicado con White en 1978, donde se ofrece un modelo original, dentro de la psicología del procesamiento de la información. En él se trata de poner de manifiesto las posibles relaciones que existen entre las diversas estructuras de la memoria con los resultados del aprendizaje. Como puede observarse en la figura 6, las habilidades intelectuales (punto fuerte de la teoría de Gagné) se integran explícitamente como un tipo particular de estructura de la memoria (4). Analizando la cuarta edición del libro Las condiciones del aprendizaje (The conditions of learning and theory of Instruction) realizada en 1985, puede observarse que, aunque se incorporan a los términos utilizados los provenientes de otros modelos de aprendizaje emparentados con la psicología del procesamiento de la información. (organizadores del aprendizaje, esquemas, conocimiento declarativo y procedimental, etc.), no se avanza en relación al modelo presentado en 1978.

figura $S$

Este es el «modelo básico del aprendizaje y la memoria», que presenta Gagné en su libro «Principios basicos del aprendizaje para la instruc ción", p. 25. Está inspirado en el modelo de la teoría del procesamiento de la información de Lindsay y Norman (1972).

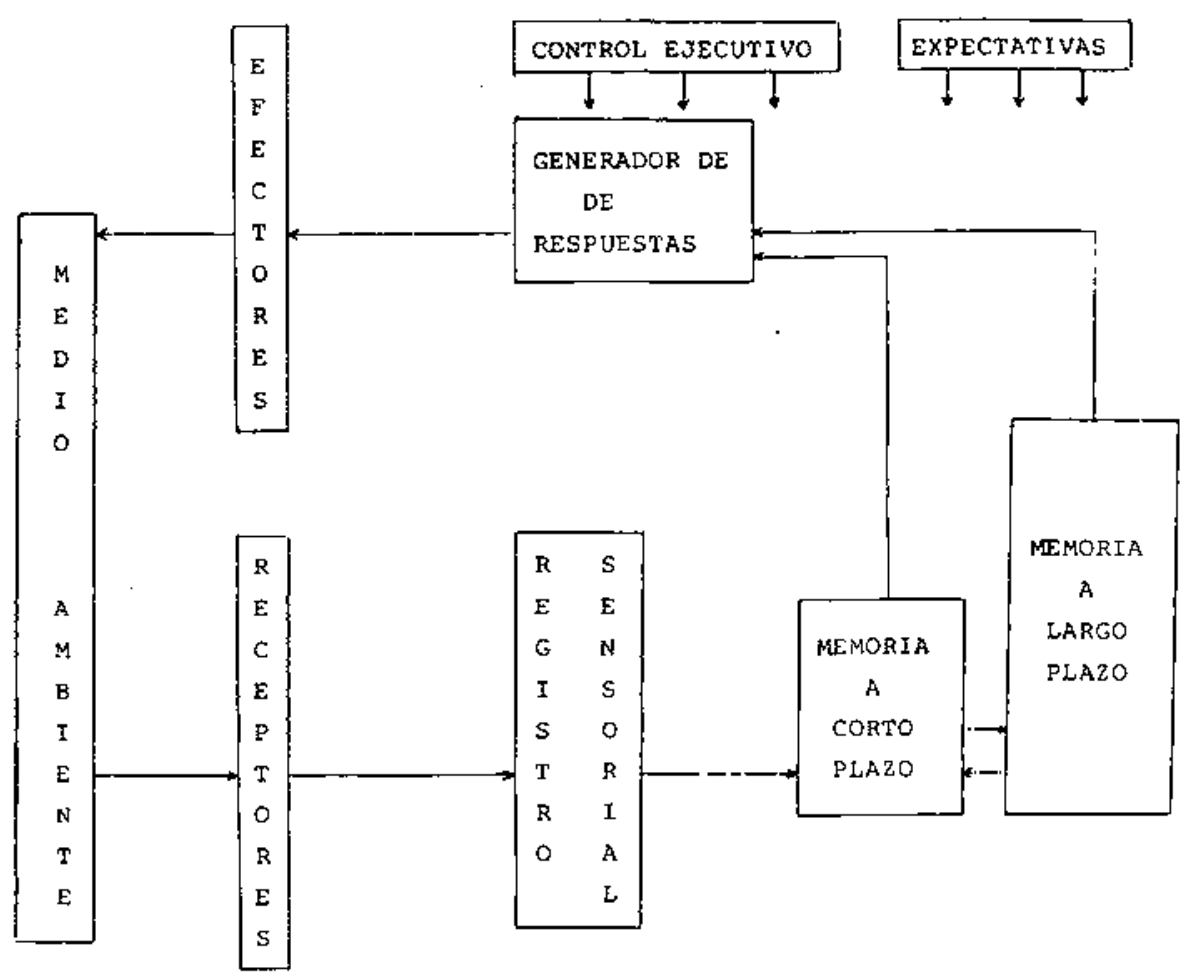


figura 6

Modelo original de Gagné y White en el que tratan de dar cuenta del aprendizaje dentro de la Psicología del Procesamiento de la Información. Obsérvese que las habilidades intelectuales forman parte de la estructura de la memoria. Las interrelaciones entre esta parte de la memoria y las otras están sin explicitar en el modelo (Gagné y White 1978, p. 195).

ESTRUCTURA DE LA MEMORIA CONDUCTA RESULTANTE

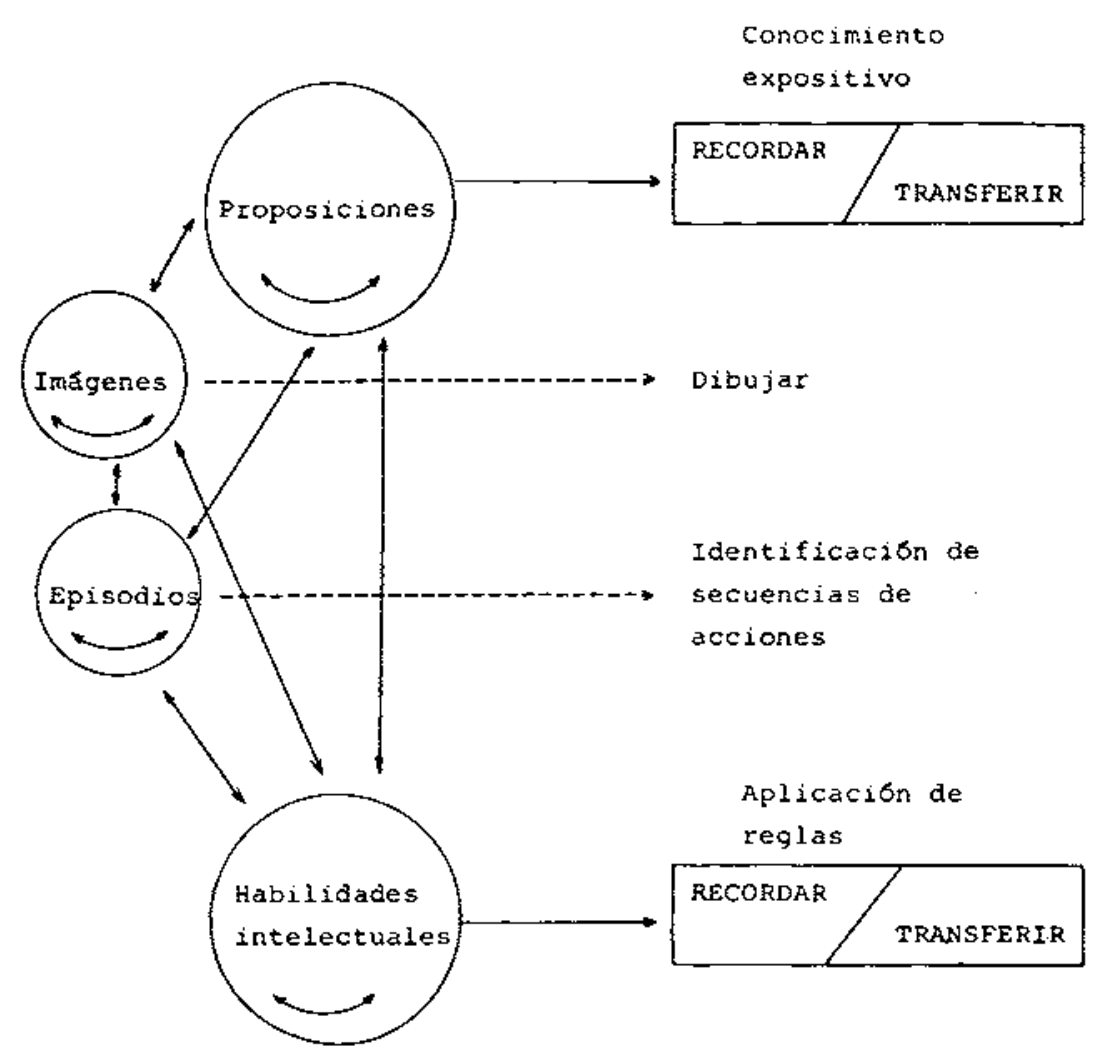

Por todo lo dicho en este apartado, puede afirmarse a modo de sintesis que el modelo gagnetiano ha evolucionado en el pensamiento de su propio autor, hasta poder ser explicado completamente dentro de un modelo más amplio de Procesamiento de la Información. Así lo ha entendido el mismo Gagné y así lo entienden otros autores cuando se refieren a su obra (Gagné y Berliner 1984, Stewart y Atkin 1982, White y Tisher 1986).

\section{4. ¿GAGNÉ EN LAS AULAS DE hOY?}

Después de analizar las críticas hechas al modelo gagnetiano, su restringido ámbito de aplicación, su evolución hacia tentativas explicativas más amplias, mucha gente se pregunta: ¿Por qué estudiar a Gagné? ¿No es un modelo superado? Si él mismo se explica a sí mismo desde un modelo de Procesamiento de la Información, ¿por qué no empezar por ahí y olvidar a Gagné?

La respuesta a todas estas preguntas es un poco arriesgada y, lamentablemente, en espera de que alguna investigación sugiera respuestas más rigurosas, solo se puede contestar desde la intuición y la experiencia del contacto con la realidad escolar. En nuestra opinión,
Gagné está vivo en nuestras aulas por dos razones muy sencillas: a) porque los profesores no necesitan saber psicología para «aplicar a Gagné», y b) porque el modelo gagnetiano "funciona".

a) Es fácil comprobar que cuando un profesor prepara bien su clase (aunque no haya estudiado nada de didáctica), lo que ha hecho en muchos casos es un intento de secuencia instructiva que sigue la lógica de los conceptos científicos que quiere ensettar. Normalmente procede desde lo que parece más fácil -desde el punto de vista lógico- hasta lo que parece más dificil, edificando sobre los conceptos más simples los conceptos más complejos. Cuando los alumnos fallan en el aprendizaje, normalmente lo atribuyen a lagunas lógicas en el proceso constructivo, al fallo en el aprendizaje de conceptos más sencillos, que se consideran básicos (prerrequisitos) para el aprendizaje de tipo superior. En la mayoría de los casos este profesor no sabe nada de Gagné, ni de sus principios de aprendizaje. Pero si alguna vez oye hablar de él, se reconocerá de alguna manera.

b) Hay profesores que hacen del diseño del tipo gagnetiano (consciente o inconscientemente realizado) un «procedimiento» para la adquisición de determinada 
habilidad intelectual. Y parece claro que el aprendizaje de procedimientos funciona para conseguir que el sujeto exhiba la conducta aprendida deseada. En la práctica, el peligro está en que el profesor caiga en la rutina (muchas veces bajo la presión de un programa que hay que terminar) de: «Tu apréndete esto, que ya Io entenderás", $y$ acepte con facilidad un aprendizaje que no implique comprensión en ámbitos en que ésta sea necesaria.

\section{Notas:}

(1) Subrayados del mismo Gagné.

(2) Traducción nuestra.

(3) Vale aqui la argumentación hecha por Herron (1978), cuando critica a Novak por su interpretación del modelo de Ausubel. Cfr. también Otero 1985.

\section{REFERENCIAS BIBLIOGRÁFICAS}

BASS, J.E. y MONTAGUE, E.J., 1972. Piaget-based sequences of instruction in Science, Science Education, Vol. 56(4), pp. 503-512.

BRIGGS, L.J., 1973a. El ordenamiento de secuencia en la instruccion. (Guadalupe: Buenos Aires). (Ed. orig: Sequencing of instruction in relation of hierarchies of competence. American Institute for Research: Pittsburgh, Filadelfia, 1968.

BRIGGS, L.J., 1973b. Manuel para el disetio de la instrucción. (Guadalupe: Buenos Aires). (Ed. orig: Handbook of procedures for the design of instruction. American Institute for Research: Pittsburgh, Filadelfia, 1970.

CASE, R. y BEREITER, C., 1984. From behaviourism to cognitive behaviourism to cognitive development: steps in the evolution of instructional design. Instructional Science, Vol. 13(2), pp. 141-158.

CAWTHRON, E.R. y ROWELL, J.A., 1978. Epistemology and Science Education, Studies in Science Education, Vol. 5. pp. 31-59.

DRIVER, R., 1982. Children's learning in Science, Educational Analysis, Vol. $4(2)$, pp. 69-79.

DRIVER, R., 1983. The pupil as scientist? (Open University Press: Milton Keynes, U.K.).

FINLEY, F.N., 1983. Science Process, Journal of Research in Science Teaching, Vol. 20(1), pp. 47-57.

GAGE, N.L. y BERLINER, D.C., 1984. Educational Psychology. (Houghton Mifflin, Co.: Boston).

GAGNE, R.M., 1962. The adquisition of Knowledge. Psychological Review. Vol. 69(4), pp. 355-365.
En todo caso, hay que reconocer que, dado el nivel de confusión que se observa con demasiada frecuencia, tanto en disenos curriculares (en nuestro país y fuera de él), como en el diseño (implícito o explícito) de las unidades didácticas particulares que el profesor sigue en la clase, no estaria mal que al menos se consiguiera una cierta coherencia lógica en los materiales y en las exposiciones, aunque sea ésta una lógica de tipo gagnetiano. Por algo hay que empezar.

(4) Es indudable la infuencia que en este modelo tiene el presentado por White un aflo antes (1977), que ya incluye integrados en un modelo de Procesamiento de la Información, las habilidades intelectuales como las describe Gagné en su obra Las condiciones del aprendizaje.

GAGNÉ, R.M., 1971. Las condiciones del aprendizaje. (Aguilar: Madrid). (Ed. orig: The conditions of learning, Holt, Rinehart \& Winston: New York, 1965).

GAGNÉ, R.M., 1975. Principios básicos del aprendizaje para la instrucción. (Diana: México). (Ed. orig.: Essential of learning for instruction. Dryden Press, 1974).

GAGNE, R.M., 1977. The conditions of learning. ( $3^{3}$ ed.). (Holt, Rinehart Winston: N.Y.).

GAGNE, R.M., 1985. The conditions of learning and Theory of Instruction ( $4^{3}$ ed.). (Holt-Saunders International Editions: Japan).

GAGNÉ, R.M. y BRIGGS, L.J., 1976. La planificación de la enseranza. (Trillas: México). (Ed, orig: Principles of instructional design. Holt, Rinetart \& Winston: N.Y., 1974).

GAGNÉ, R.M. y WHITE, R.T., 1978. Memory structures and learning outcomes, Review of Educational Researh, Vol. 48(2), pp. 187-222.

GAGNE, R.M. y DICH, W., 1983. Instructional Psychology, Ann. Rev. of Psychology, Vol. 34, pp. 261-295.

GUTIERREZ, R, 1987. Psicologfa y aprendizaje de las Ciencias. El modelo de Ausubel, Enseñanza de las Ciencias, Vol. $5(2)$, pp. 118-128.

HARTLEY, J. y DAVIES, 1.K., 1976. Preinstructional strategies: the role of pre-test, behavioral objectives, and advance organizers, Review of Educational Research, Vol. 46(2), pp. 239-265.

HERRON, J.D., 1978. Role of learning and development: critique of Novaks comparation of Ausubel and Plaget, Science Education, Vol. 62(4), pp. 593-605. 
HEWSON, P.W., 1981. A conceptual change approach to learning science, European Journal of Science Education, Vol. 3(4), pp. 383-396.

JONES, H.L., y RUSSEL, J.M., 1979. Hierarchical learning paradigm, Journal of Research in Science Teaching, Vol. 16(6), pp. 489-499.

KELLET, N.C., WEST, L.H.T. y WOODRUFF, C., 1977. Meastures of meaningful learning of intellectual skills, $R e$ seach in Science Education, Vol. 7, pp. 33-40.

NESBIT, J.E. y HUNCA, S., 1987. A method for sequencing instructional objectives which minimizes memory load, Instructional Science, vol. 16(2), pp. 137-150.

NOVAK, J., 1982. Teoría y práctica de la Educación. (Alianza: Madrid). (Ed. orig: A theory of Education. Cornell University Press: Ithaca, 1977).

OLAREWAJU, A.O., 1987. Relative effects of hierarchical versus non-hierarchical learning tasks on students' achievement in Biology, Res. in Science and Tech. Education. Vol. 5(1), pp. 177-124.

OTERO, J., 1985. El aprendizaje de los conceptos cientifi$\cos$ en los niveles medio y superior de la enseñanza, $R e$ vista de Educación, Vol. 278, pp. 39-66.

PHILLIPS, D.C. y KELLY, M.E., 1975. Hierarchical theories of development in Education and Psychology, Harvard Educational Review, Vol. 45(3). pp. 351-375.

POSNER, G.J. y STRIKE, K.A., 1976. A categorization scheme for principles of sequencing content, Review of Education Research, Vol. 46(4), pp. 655-690.

POSNER, G.J., STRIKE, K.A., HEWSON, P.W. y GERTZOG, W.A., 1982. Accomodation of scientific conception: Toward a theory of conceptual change. Science Education, Vol. 66(2), pp. 211-227.

POZO, J.I., 1987. Aprendizaje de la Ciencia y pensamiento causal. (Visor: Madrid).
RAVEN, R., 1967-68. The development of the concept of momentum in Primary school children, Journal of Research in Science Teaching, Vol. 5(3), pp. 216-233.

RAVEN, R., 1972. The development of the concept of acceleration in elementary school children, Journal of Research in Science Teaching, Vol. 9(3), pp. 206-212.

RESNICK, I., 1983. Vers une theorie cognitive de la didactique, en Giordan, A. y Martinand, J.L. (eds.), Quels types de recherche pour rénover l'éducation en sciences experimentales?, $5^{2}$ Jornadas Internacionales sobre la Educación Cientifica, Chamonix, pp. 137-149.

SOULSBY, D., 1975. Gagné's hierarchical theory of Iearning: some conceptual difficulties, Journal of Curr. Studies, Vol, 7(2), pp. 123-132.

STEWART, J.H. y ATKIN, J.A., 1982. Information Processing Psychology: a promising paradigm for research in science teaching, Journal of Research in Science Teaching, vol. 19(4), pp. 321-332.

TOMLINSON, P., 1984. Psicología Educativa. (Pirámide: Madrid). (Ed. orig: Understanding teaching. Interactive Educational Psychology. (Mac Graw-Hill: London, 1981).

WEST, L.H. y FENSHAM, J., 1974. Prior knowledge and the learning of science: a review of Ausubel's theory of this process, Studies in Science Education, Vol. 1, pp. 61-81.

WHITE, R.T., 1974. A model for validation of learning hierarchies, Journal of Research in Science Teaching, Vol. $11(1)$, pp. 1-3.

WHITE, R.T., 1977. A model of cognitive processes, $R e$ search in Science Education, Vol. 7, pp. 25-35.

WHITE, R.T. y TISHER, R.P., 1986. Research on Natural Sciences, en Wittrock, M.C. (ed.), Handbook of Research on Teaching. (AERA. MacMilian: N.Y.), pp. 874-905. 